\title{
Fermented dairy food and CVD risk
}

\author{
Linda C. Tapsell* \\ School of Medicine, Smart Foods Centre, Illawarra Health and Medical Research Institute, University of Wollongong, \\ Wollongong, NSW 2522, Australia
}

(Submitted 4 March 2014 - Final revision received 6 June 2014 - Accepted 27 June 2014)

\section{Abstract}

Fermented dairy foods such as yoghurt and cheese are commonly found in the Mediterranean diet. Recent landmark research has confirmed the effect of the Mediterranean diet on reducing the CVD risk, but the relative contributions of fermented dairy foods have not been fully articulated. The present study provides a review of the relationship between fermented dairy foods consumption and CVD risk in the context of the whole diet. Studies show that people who eat healthier diets may be more likely to consume yoghurt, so there is a challenge in attributing separate effects to yoghurt. Analyses from large population studies list yoghurt as the food most negatively associated with the risk of weight gain (a problem that may lead to CVD). There is some suggestion that fermented dairy foods consumption (yoghurt or cheese) may be associated with reduced inflammatory biomarkers associated with the development of CVD. Dietary trials suggest that cheese may not have the same effect on raising LDL-cholesterol levels as butter with the same saturated fat content. The same might be stated for yoghurt. The use of different probiotic cultures and other aspects of study design remain a problem for research. Nevertheless, population studies from a range of countries have shown that a reduced risk of CVD occurs with the consumption of fermented dairy foods. A combination of evidence is necessary, and more research is always valuable, but indications remain that fermented dairy foods such as cheese and yoghurt are integral to diets that are protective against CVD.

\section{Key words: Dairy foods: CVD: Nutrition}

The landmark Prevención con Dieta Mediterránea (PREDIMED) study confirmed that the Mediterranean diet reduces the risk of CVD by about $30 \%{ }^{(1)}$. The diet is a composite of foods, so understanding the contribution of each food group is important. The interdependence between individual foods, their significant nutritional components ${ }^{(2)}$ and the total diet will need to be considered. There is a wide range of foods with different compositions in the dairy products category, but fermented dairy foods such as yoghurt and cheese are commonly found in the Mediterranean $\operatorname{diet}^{(3)}$. The relative contributions of fermented dairy foods in reducing the CVD risk have not been fully articulated in the scientific literature.
The association between fermented dairy foods consumption and reduced cardiovascular risk appears in a number of ways. For example, observational research has shown that yoghurt consumption is negatively associated with weight gain, and cheese has a more neutral effect ${ }^{(4)}$. Other studies have demonstrated that fermented dairy products have anti-hypertensive effects $^{(5)}$, and a number of small trials have shown that cheese intake does not have the same effect on cholesterol levels compared to that of butter ${ }^{(6)}$. Thus, the consumption of fermented dairy foods, in particular yoghurt and cheese, may contribute substantively to the prevention of CVD risk. The present study provides a review of the association between

Abbreviations: DGAI, Dietary Guidelines Adherence Index; EPIC, European Prospective Investigation into Cancer and Nutrition; HR, hazard ratio; PREDIMED, Prevención con Dieta Mediterránea.

*Corresponding author: L. C. Tapsell, fax +6124221 4844, email ltapsell@uow.edu.au

Publication of these papers was supported by unrestricted educational grants from Federación Española de Sociedades de Nutrición, Alimentación y Dietética (FESNAD), International Nut and Dried Fruit Council (INC), International Union of Nutritional Sciences (IUNS), Fundación Iberoamericana de Nutrición (FINUT), Centro de Investigación Biomédica en Red de la Fisiopatología de la Obesidad y Nutrición (CIBERobn) and Centro Interuniversitario di Ricerca sulle Culture Alimentari Mediterranee (Ciiscam). The papers included in this supplement were invited by the Guest Editors and have undergone the standard journal formal review process. They may be cited. The Guest Editors declare that Salas-Salvadó is a nonpaid member of the World Forum for Nutrition Research and Dissemination of the International Nut and Dried Fruit Council. Angel Gil is President of the Fundación Iberomericana de Nutrición, which is a non-paid honorary position. Lluis Serra-Majem is the President of the Scientific Committee of the Mediterranean Diet Foundation and Scientific Director of the CIISCAM (Centro Interuniversitario di Ricerca sulle Culture Alimentari Mediterranee), Universita La Sapienza di Roma which are both non-paid, honorary positions. Goretti Guasch is the Executive Director and Member of the Executive Committee of the International Nut and Dried Fruit Council, which is a paid position. Mònica Bulló declares no conflict of interest. 
fermented dairy foods consumption and CVD risk in the context of the whole diet.

\section{Fermented dairy foods, body weight and CVD risk}

Excess body weight is a risk factor for CVD and excessive energy intake from food is a significant cause of obesity. The consumption of fermented dairy foods may have a primary preventive effect on CVD by supporting the development of a healthy body weight. One of the proposed mechanisms for this effect is through supporting a healthy gut microbiota. Animal model experiments suggest that a 'leaky' gut wall is implicated in the development of obesity and associated basal inflammation ${ }^{(7)}$, adding to the understanding of the links between inflammation and central obesity with the development of $\mathrm{CVD}^{(8)}$. Much of the research on this topic has focused on yoghurt consumption. In a recent review, Marette \& Picard-Deland ${ }^{(9)}$ argued that the early introduction of yoghurt into the diets of children was important in establishing a microbial community that supports long-term health. More research is needed, but in the first instance, yoghurt can deliver essential nutrients with high bioavailability and relatively low energy density.

The energy value of yoghurt may be a problem if the energy content of the diet is not managed, particularly with high-fat varieties and with added ingredients such as sugar. This problem was demonstrated in a trial from Spain involving students supplemented with whole-fat or low-fat milk and yoghurt. In the present study, supplementation with wholefat products increased weight significantly compared to the low-fat supplements $(1.2 \mathrm{~kg}, 95 \% \text { CI } 0.5,1.8 ; P=0.007)^{(10)}$. Here, forty-five students were asked to consume usual diets (substituting dairy foods) and additional efforts were not made for reducing the energy intake. Thus, the 'free-living' nature of the study demonstrated how the fat content of the dairy supplements acted as a passive source of additional energy when other parts of the diet were not considered. Likewise, providing advice to consume low-fat dairy products may not achieve the intent of reduced energy intakes. In one analysis of trial data, we found females changed their dairy choices to consume higher carbohydrate dairy foods (notably fruited yoghurt), creating no energy deficit, whereas males simply reduced dairy food intake with negative consequences for overall nutritional intakes ${ }^{(11)}$. A similar finding emerged from a 24-week randomised controlled trial involving ninetythree families with 4-13-year-old children. In this case, advice to consume low-fat dairy products achieved reductions in saturated fat intake, but not energy intakes or body weight ${ }^{(12)}$. Systematic reviews acknowledge the complexity of the relationship between dairy foods consumption and body weight, particularly given the range of different foods in the category ${ }^{(13,14)}$. Thus, it was not surprising that a metaanalysis of randomised controlled trials failed to show weight loss effects of dairy foods consumption in the absence of energy restriction and that significant heterogeneity was found in the evidence base ${ }^{(14)}$.

Observational studies may provide a more consistent picture but are limited by residual confounding. In a cross-sectional analysis of data from two cohorts related to the Framingham Study, yoghurt consumers were found to have higher scores for the Dietary Guidelines Adherence Index (DGAI) and higher intakes of key micronutrients than non-consumers of yoghurt ${ }^{(15)}$. $\mathrm{K}$ and fibre intakes were significantly higher for high yoghurt consumers compared to low yoghurt consumers ( $P_{\text {trend }}<0.001$ and $P_{\text {trend }}<0.002$ respectively, adjusted for covariates and DGAI score). This places yoghurt consumption in the context of a healthy diet, from which benefits are likely to occur. The challenge lies in accounting for the contribution of yoghurt itself.

A much larger study was conducted with data combined from three prospective studies of 22557 men and 98320 women in the USA examining the association between changes in food/beverage consumption and body weight ${ }^{(4)}$. In this analysis, yoghurt consumption was negatively associated with a 4-year weight gain $(-0.82,95 \% \mathrm{CI}-0.99$, $-0.67 \mathrm{lb} ; P<0.001$ ), adjusted for age, baseline BMI, and numerous lifestyle and other dietary factors. Both the Framingham study and the present study implicate yoghurt consumption as a significant contributory factor in delivering effects of a healthy diet.

As suggested earlier, one of these effects may be ameliorating the impact of inflammation associated with obesity. For example, in a cross-sectional analysis of the ATTICA study (n 3042 Greek adults), inverse associations were found between markers of inflammation (C-reactive protein, IL-6 and $\mathrm{TNF}-\alpha$ ) and consumption of dairy products in general ${ }^{(16)}$. Compared to those consuming fewer than eight servings per week, significant reductions were found between those consuming between eleven and fourteen serves per week $(P<0.05)$ and the differences were even greater for those consuming more than fourteen servings per week $(P<0 \cdot 01)$. Although the analysis focused on dairy foods as a group, a greater proportion of the cohort reported consumption of fermented dairy foods such as feta cheese (93\%), hard yellow cheese (92\%) and low-fat yoghurt (50\%) compared to low-fat milk (46\%).

More focus on fermented dairy foods is needed in order to expose the individual food effects from within the food category $^{(17)}$. For example, in a 3-week crossover study comparing the effects of dairy foods categorised as low-fat (milk/yoghurt), fermented (yoghurt/cheese) or non-fermented (butter/cream/ice cream), the concentrations of IL-6 were significantly lower on the low-fat or fermented dairy diet than on the non-fermented dairy diet $(P<0.05)^{(17)}$. The authors cautioned that a number of biomarkers should be considered not least because the disease itself has many facets. A single biomarker is insufficient for addressing the CVD risk, rather multiple biomarkers and studies with clinical endpoints are required ${ }^{(18)}$.

In summary, fermented dairy products have the potential to assist in maintaining a healthy weight, support the integrity of the gut and ameliorate the effects of basal inflammation to reduce the CVD risk. For effects to be realised, energy intakes need to be compatible with a healthy weight. 


\section{Fermented dairy foods, lipids and CVD risk}

While the fat content of dairy foods may be at issue for weight management, evidence linking dietary SFA to high LDL levels has implicated dairy food consumption with an increased risk of CVD. Cheese is a fermented dairy food with a high SFA content. A recent review on dairy foods and CVD risk ${ }^{(19)}$ examined nine trials addressing this issue and concluded that cheese may not increase LDL-cholesterol to the same extent as butter compared with a lower SFA diet ${ }^{(19)}$. In the same review, fifteen studies were considered on the effects of fermented milk and yoghurt. These produced little evidence that conventional starter cultures (Lactobacillus bulgaricus and Streptococcus thermophilus) lower LDLcholesterol or TAG or raise HDL-cholesterol. The main problem with the evidence base was the choice of controls and presence of confounding. In addition, the use of different probiotic bacterial strains made comparisons difficult ${ }^{(19)}$. Others have arrived at a similar conclusion ${ }^{(6)}$ arguing the need to control for energy and macronutrient intakes, to determine appropriate bacterial concentrations and to consider the blood lipid status of subjects.

Whether effects on lipids translate to reductions in the CVD risk can be considered with studies of disease end points. At least one study involving a dose-response meta-analysis has confirmed no significant associations between the consumption of dairy food products (per $200 \mathrm{~g}$ daily) and $\mathrm{CHD}^{(20)}$. In their review of epidemiological studies, Huth \& $\operatorname{Park}^{(19)}$ found no evidence of a consistent association between intakes of dairy products generally or cheese specifically, and increased risk of CVD. Given the range of foods in the dairy category, variations in total diet and other lifestyle/environmental factors, the problem of residual confounding must be considered. In another review, Gibson et al. ${ }^{(21)}$ argued that problems also include bias in the study design, in particular the lack of adjustments for socio-economic and educational factors.

It is possible that yoghurt consumption may interfere with cholesterol synthesis (via increased SCFA) or reduced reabsorption of bile acids, but studies are required to confirm the hypothesis ${ }^{(6)}$. Different bacterial strains appear to have different cholesterol-lowering properties, but the bacteria need to be able to survive the gut and colonise the intestine (i.e. have a probiotic effect). Reviews have argued the antihypertensive effects of milk fermented with Lactobacillus belveticus that may be due to the angiotensin-converting enzyme inhibition from peptides ${ }^{(5)}$, and the effect of cheese may be a response to the Ca content or peptides produced during fermentation ${ }^{(5)}$.

New technologies provide other ways to investigate possible effects of dairy products on reducing the CVD risk. In the main Syracuse cohort ( $n$ 587), arterial stiffness was assessed with measures of carotid-femoral pulse wave velocity and pulse pressure ${ }^{(22)}$. Statistically significant negative linear trends were observed for both these variables with an increasing dairy food intake $(P<0 \cdot 01)$. No associations were detectable for between carotid-femoral pulse wave velocity and different categories of dairy foods such as milk, cheese, and yoghurt $(P>0.30)$, but the sample was small and the intakes were highly variable. Milk was most commonly consumed, then cheese (two to four times per week), and yoghurt less frequently. In contrast, a study of $>1500$ older women in Perth, Australia, showed a negative association between yoghurt consumption and common carotid artery intima-media thickness (CCA-IMT) (standardised $\beta=-0.075, P=0.015)$. This was not the case for cheese, milk or total dairy food intake $(P>0.05)$. The difference between those who consumed $>100 \mathrm{~g} / \mathrm{d}$ compared to those with lower consumptions was still significant even after adjustments $\left(C C A-\mathrm{IMT}_{\mathrm{adj}}=-0.023 \mathrm{~mm}, P=0.003\right)^{(23)}$. Clinical trials are required to confirm the apparent protective effect of yoghurt consumption on intima media thickening of the carotid artery, at least in women.

In summary, components in fermented dairy foods may help to reduce the risk of CVD by interfering with cholesterol pathways $^{(6)}$ or they may have antihypertensive properties ${ }^{(5)}$, and possibly more local positive effects on the arterial wall $^{(23)}$. More randomised controlled trials are required as associations do not prove causality ${ }^{(24)}$, but the picture is becoming clearer. As with the weight management, the contribution of fermented dairy products to reduced CVD risk is likely observed in the context of a healthy diet.

\section{Fermented dairy food and CVD risk in different dietary contexts}

The emphasis on positioning individual foods in healthy diets, rather than studying them in isolation, is a major shift in research, but it allows for more natural exposure of effects. There are limited reports in the scientific literature on healthy diets, with the exception of the Mediterranean diet. Indeed, there is global recognition of the Mediterranean diet within United Nations Educational, Scientific and Cultural Organization's Intangible Cultural Heritage list, expanding the appreciation of the diet to that of local and global values ${ }^{(25)}$ The PREDIMED study ${ }^{(1)}$ provided a sound scientific basis for setting the Mediterranean diet as a platform for quantifying the impact of diet on reducing the risk of CVD. The next step is confirming the types of foods that together constitute protective effects.

Populations consume foods available within regions. Given the evidence on the effect of the Mediterranean diet, studies from Europe provide a good starting point for reviewing the protective effects of fermented dairy foods. In a nested cohort study ( $n$ 16835) of the European Prospective Investigation into Cancer and Nutrition (EPIC)-InterAct Study (eight of ten EPIC countries), an inverse association was found between the risk of type 2 diabetes (a risk factor for CVD) and the consumption of cheese (hazard ratio (HR) 0.88, $95 \%$ CI $0.76,1.02 ; P_{\text {trend }}=0.01$ ) and fermented dairy food (yoghurt and fermented milk) (HR 0.88, 95\% CI 0.78, 0.99; $\left.P_{\text {trend }}=0.02\right)^{(26)}$. The strength of this analysis was that it cuts across different populations in the European region. The profile of dairy food consumption in the Netherlands might explain the small inverse association between fermented full-fat dairy food intakes and all-cause mortality in the Netherlands cohort study (NCLS) cohort ( $n$ 120, 852) $)^{(27)}$. 
In contrast, data from the EPIC-Norfolk cohort in the UK ( $n$ 4000) produced an association between low-fat fermented dairy product consumption and reduced risk of type 2 diabetes (HR 0.76, 95\% CI 0.60, 0.99; $P_{\text {trend }}=0.049$ ), and more specifically for yoghurt intake (HR 0.72, 95\% CI 0.55 , $\left.0 \cdot 95 ; P_{\text {trend }}=0 \cdot 017\right)^{(28)}$

It may be that the impact of fermented dairy foods and yoghurt is more pronounced in different dietary contexts. An analysis of a subset of data in the Malmo Diet and Cancer cohort ( $n$ 24445) found that the consumption of dairy products overall was inversely associated with CVD risk $\left(P_{\text {trend }}=0.05\right)^{(29)}$. Fermented milk was the only product with a statistically significant negative relationship with CVD risk (about 15\%) $\left(P_{\text {trend }}=0.003\right)$. A decreased CVD risk was found with cheese intake in women $\left(P_{\text {trend }}=0.03\right)$ but not in men. These findings were similar to those from an analysis of the Swedish Mammography Cohort study ( $n$ 33636; 1997 subset $)^{(30)}$. This study found that total dairy intake was inversely associated with the risk of myocardial infarction $\left(\mathrm{HR}_{\mathrm{adj}} 0.77,95 \% \mathrm{CI} 0.63,0.95\right)$. Total cheese intake was inversely associated with myocardial infarction risk (HR $0 \cdot 74$, 95\% CI 0.60, 0.91). Importantly, the authors of this analysis stressed the need to differentiate between different foods in the dairy category, and to not treat all dairy foods as the same. As they found differences in results for butter consumption depending on its use in the diet, they also acknowledged the limitations for research inherent in the cultural use of dairy foods ${ }^{(30)}$.

To take this discussion further, studies outside of Europe may be informative. From Japan, factor analysis using data from the Osaka National Health Insurance (NIH) cohort found a lower CVD risk with what was termed the Japanese pattern (fish, seaweed, vegetables, fruit and green tea) but it was associated with a high $\mathrm{Na}$ intake and greater risk of hypertension. An increased risk was found with the 'animal food' pattern, but a third pattern defined by high dairy food, high fruit and vegetables and low alcohol intake was not associated with increased CVD risk ${ }^{(31)}$. In an even more diverse context, in remote Canada, no association was found between dairy food intake and the incidence of CVD in a cross-sectional study of fourteen villages of the Nunavik ${ }^{(32)}$. This was not surprising, as the study was small ( $n$ 543), and the intake of dairy food was small (120-290 g/d). Even so, reviewing research from various regions indicates that the effects of fermented dairy foods are likely dependent on the degree of dietary exposure alongside other food and environmental factors.

In summary, the protective effect of the Mediterranean diet on CVD risk has been confirmed. Evidence of associations between the consumption of fermented dairy products and reduced CVD risk has been found in the concept of a healthy diet. There is some variation in findings across populations and research designs can be improved upon, but there is enough to go on. Part of the problem is the somewhat intangible nature of studying effects of nutrients, foods and whole diets simultaneously, but the recognition of the Mediterranean diet as a concept throws light on the task. The implications for translating this knowledge for a broader benefit to the global population present new and exciting challenges.

\section{Conclusion}

The association between fermented dairy foods consumption and CVD risk rests on the study of healthy diets and individual foods. Research to date strongly implicates fermented dairy products as the products of choice in demonstrating effects. Yoghurt and cheese are traditionally part of the Mediterranean diet, the protective effects of which are confirmed.

Treating the risk associated with saturated fat intakes and blood cholesterol in the isolation of food sources is now seriously challenged ${ }^{(18,33,34)}$. Expert panels have noted that while there is evidence for replacing SFA with PUFA in the diet, effects of single foods cannot be judged solely on their SFA content ${ }^{(18,33)}$. This is because different SFA contents may have different effects, and the foods themselves deliver multiple nutrients ${ }^{(18)}$. The concept of food synergy, in which the sum of the parts is greater than the individual ingredient, is an important new consideration ${ }^{(35)}$. There is a need to differentiate between dairy products because of the variation in the content ${ }^{(33)}$. Moderate amounts of fermented dairy foods play a significant role in dietary patterns that protect against CVD but more targeted research is required. More needs to be known on the type of food, in particular cheese and yoghurt. The overall diet remains an important factor in terms of energy and nutrient balance, food variety and amounts of foods consumed. A combination of evidence is necessary ${ }^{(18)}$, and more research across various regions will assist this, but indications remain that fermented dairy foods such as yoghurt and cheese are an integral part of diets that are protective against CVD.

\section{Acknowledgements}

No funding support was provided for the preparations of this manuscript.

There is no conflict of interest to declare.

\section{References}

1. Estruch R, Ros E, Salas-Salvadó J, et al. (2013) Primary prevention of cardiovascular disease with a Mediterranean diet. $N$ Engl J Med 368, 1279-1290.

2. Saura-Calixto F \& Goni I (2009) Definition of the Mediterranean diet based on bioactive compounds. Crit Rev Food Sci Nutr 49, 145-152.

3. Vasilopoulou E, Dilis V \& Trichopoulou A (2013) Nutrition claims: a potentially important tool for the endorsement of Greek Mediterranean traditional foods. Medit J Nutr and Metabolism 6, 105-111.

4. Mozaffarian D, Hao T, Rimm EB, et al. (2011) Changes in diet and lifestyle and long-term weight gain in women and men. $N$ Engl J Med 364, 2392-2404

5. Tholstrup $\mathrm{T}$ (2006) Dairy products and cardiovascular disease. Curr Opin Lipidol 17, 1-10.

6. Nestel PJ (2008) Effects of dairy fats within different foods on plasma lipids. J Am Coll Nutr 27, 735S-740S.

7. Lam YY, Mitchell AJ, Holmes AJ, et al. (2011) Role of the gut in visceral fat inflammation and metabolic disorders. Obesity 19, 2113-2120.

8. Grundy SM (2012) Pre-diabetes, metabolic syndrome, and cardiovascular risk. J Am Coll Cardiol 59, 635-643. 
9. Marette A \& Picard-Deland E (2014) Yogurt consumption and impact on health: focus on children and cardiometabolic risk. Am J Clin Nutr 99, 1243S-1247S.

10. Alonso A, Zozaya C, Vázquez Z, et al. (2009) The effect of low-fat versus whole-fat dairy product intake on blood pressure and weight in young normotensive adults. J Hum Nutr Diet 22, 336-342.

11. Nolan-Clark D, Neale E, Probst Y, et al. (2013) Dietary consequences of recommending reduced-fat dairy products in the weight-loss context: a secondary analysis with practical implications for registered dietitians. I Acad Nutr Diet 113, 452-458.

12. Hendrie GA \& Golley RK (2011) Changing from regular-fat to low-fat dairy foods reduces saturated fat intake but not energy intake in 4-13-y-old children. Am J Clin Nutr 93, $1117-1127$

13. Kratz M, Baars $T$ \& Guyenet S (2013) The relationship between high-fat dairy consumption and obesity, cardiovascular, and metabolic disease. Eur J Nutr 52, 1-24.

14. Chen M, Pan A, Malik VS, et al. (2012) Effects of dairy intake on body weight and fat: a meta-analysis of randomized controlled trials. Am J Clin Nutr 96, 735-747.

15. Wang H, Livingston KA, Fox CS, et al. (2013) Yogurt consumption is associated with better diet quality and metabolic profile in American men and women. Nutr Res 33, 18-26.

16. Panagiotakos DB, Pitsavos CH, Zampelas AD, et al. (2013) Dairy products consumption is associated with decreased levels of inflammatory markers related to cardiovascular disease in apparently healthy adults: the ATTICA study. J Am Coll Nutr 29, 357-364.

17. Nestel PJ, Mellett N, Pally S, et al. (2013) Effects of low-fat or full-fat fermented and non-fermented dairy foods on selected cardiovascular biomarkers in overweight adults. Br J Nutr 110, 2242-2249.

18. Astrup A (2014) Yogurt and dairy product consumption to prevent cardiometabolic diseases: epidemiologic and experimental studies. Am J Clin Nutr 99, 1235S-1242S.

19. Huth PJ \& Park KM (2012) Influence of dairy product and milk fat consumption on cardiovascular disease risk: a review of the evidence. Adv Nutr 3, 266-285.

20. Soedamah-Muthu SS, Verberne LD, Ding EL, et al. (2012) Dairy consumption and incidence of hypertension: a dose-response meta-analysis of prospective cohort studies. Hypertension 60, 1131-1137.

21. Gibson RA, Makrides M, Smithers LG, et al. (2009) The effect of dairy foods on CHD: a systematic review of prospective cohort studies. Br J Nutr 102, 1267-1275.

22. Crichton GE, Elias MF, Dore GA, et al. (2012) Relations between dairy food intake and arterial stiffness: pulse wave velocity and pulse pressure. Hypertension 59, 1044-1051.
23. Ivey KL, Lewis JR, Hodgson JM, et al. (2011) Association between yogurt, milk, and cheese consumption and common carotid artery intima-media thickness and cardiovascular disease risk factors in elderly women. Am J Clin Nutr 94, 234-239.

24. Mensink RP (2006) Dairy products and the risk to develop type 2 diabetes or cardiovascular disease. Int Dairy $J \mathbf{1 6}$ 1001-1004.

25. Reguant-Aleix J, Arbore MR, Bach-Faig, et al. (2009) Mediterranean diet: an intangible cultural heritage. Pub Health Nutr 12, 1591-1594

26. Sluijs I, Forouhi NG, Beulens JWJ, et al. (2012) The amount and type of dairy product intake and incident type 2 diabetes: results from the EPIC-InterAct Study. Am J Clin Nutr 96, 382-390.

27. Goldbohm RA, Chorus AMJ, Garre FG, et al. (2011) Dairy consumption and 10-y total and cardiovascular mortality: a prospective cohort study in the Netherlands. Am J Clin Nutr 93, 615-627.

28. O'Connor LM, Lentjes MAH, Luben RN, et al. (2014) Dietary dairy product intake and incident type 2 diabetes: a prospective study using dietary data from a 7-day food diary. Diabetologia 57, 909-917.

29. Sonestedt E, Wirfält E, Wallström P, et al. (2011) Dairy products and its association with incidence of cardiovascular disease: the Malmö diet and cancer cohort. Eur J Epidemiol 26, 609-618.

30. Patterson E, Larsson SC, Wolk A, et al. (2013) Association between dairy food consumption and risk of myocardial infarction in women differs by type of dairy food. $J$ Nutr 143, 74-79.

31. Shimazu T, Kuriyama S, Hozawa A, et al. (2007) Dietary patterns and cardiovascular disease mortality in Japan: a prospective cohort study. Int J Epidemiol 36, 600-609.

32. Ferland A, Lamarche B, Château-Degat ML, et al. (2011) Dairy product intake and its association with body weight and cardiovascular disease risk factors in a population in dietary transition. J Am Coll Nutr 30, 92-99.

33. German JB, Gobson RA, Krauss RM, et al. (2009) A reappraisal of the impact of dairy foods and milk fat on cardiovascualr disease risk. Eur J Nutr 48, 191-203.

34. Griffin BA (2011) Dairy, dairy, quite contrary: further evidence to support a role for calcium in counteracting the cholesterol-raising effect of SFA in dairy foods. Br J Nutr 105, 1713-1714.

35. Jacobs DR, Gross MD \& Tapsell LC (2009) Food synergy: an operational concept for understanding nutrition. Am J Clin Nutr 89, 1S-6S 